\title{
ARMAND BOREL AS A MENTOR
}

\author{
LIZHEN JI*
}

I have the honor of being the last collaborator of Professor Armand Borel. I have learned many things from him in the last six years of his life about how to do and write mathematics, how to organize summer schools and conferences, how to keep good health and many other things. It is really sad to write this article in memory of him.

1. How I met him. I first saw Professor Borel when I visited the Institute for Advanced Study (IAS) in 1994-1995 as a member. He looked very serious to me. Though I had been interested in arithmetic groups and locally symmetric spaces, I did not ask or talk to him about mathematics even once in this whole year. In fact, I had heard of several stories about how difficult it was to start conversations with him, and I did not try to ask him anything. During the conference in honor of Langlands 60th birthday at IAS in 1997, I mustered my courage to knock at his office door. I asked him whether I could talk to him about compactifications, and he nodded his head. This first mathematical conversation lasted over two hours. He told me that he run a seminar on compactifications of symmetric spaces at IAS in 1988 and showed me some of his lecture notes. These notes discussed the Oshima compactification and variants, and complemented my interests and work about compactifications of symmetric spaces. Near the end of that conversation, we decided to work on joint projects on compactifications.

2. How the joint projects started. In fact, in a joint book Compactifications of symmetric spaces with Y.Guivarch and J.C.Taylor, we tried to discuss many other compactifications of symmetric spaces besides the Martin compactification. For example, the first noninductive description of the Karpelevic compactification was given, and many other characterizations of the maximal Satake compactifications are also given. But we were not aware of the Oshima compactification, the Oshima-Sekiguchi compactification, and the wonderful compactification of symmetric varieties by De Concini and Procesi, which were discussed in Prof. Borel's notes. In the notes, he also raised the question whether an analogue of the Oshima compactification exists for locally symmetric spaces. This naturally leads one to study compactifications of both symmetric and locally symmetric spaces simultaneously.

After returning from IAS, we corresponded frequently via email. He always responded promptly to email. To make the discussions easier, he invited me to visit IAS in November 1997 for a month. Every morning, I stopped by him office and we discussed about compactifications. In the joint book with Guivarch and Taylor, we constructed a dual-cell compactification using the Weyl chamber decomposition, and used it to relate the maximal Satake compactification to the spherical Tits building. The group action on the symmetric space can be extended to the dual-cell compactification, but the continuity of the extended action was proved via identification with other compactifications such as the Martin compactification at the bottom of the spectrum or the maximal Satake compactification. A natural question was to give a direct proof of the continuity of the extended action on the dual cell compactification without using other compactifications which support a continuous group action.

\footnotetext{
*Department of Mathematics, University of Michigan, Arbor, MI 48109, USA (lji@umich.edu).
} 
The idea of using a modified approach of the Borel-Serre compactification of locally symmetric spaces to construct compactifications of symmetric spaces was first realized during that time. For compactifications of locally symmetric spaces associated with arithmetic groups, the boundary components are naturally associated with parabolic subgroups defined over rational numbers, and the reduction theory of arithmetic groups plays a crucial role. The idea is that if one uses parabolic subgroups defined over the real numbers as well, then a suitable reduction theory for real parabolic subgroups will allow one to construct compactifications of symmetric spaces following the method for locally symmetric spaces, hence uniformizing these two types of compactifications. It also occurred to us that the Oshima compactification should be glued up from the maximal Satake compactification, and the Borel-Serre compactification can also be self-glued into a closed manifold, the desired analogue of the Oshima compactification. Though the doubling of manifolds with boundary is well-known, there is no general reference about self-gluing of manifolds with corners.

During this visit, Prof. Borel told me that he started to swim regularly in his thirties. He also told me that besides swimming, he did weight lifting in order to give every muscle enough exercise. Inspired by him, I started to swim regularly, though I did not follow his suggestion fully by combining swimming with weight lifting. This is one of the most useful things I have learned from him.

One day we went to the cafeteria at IAS, which is famous for its quality food. I commented in a teasing way that the food is so good, and it is no wonder that people wanted to come to visit IAS. He replied very seriously that he hoped that people came for mathematics, not just for the food.

In 1998, Melrose came to Ann Arbor to give a colloquium talk. He emphasized to me that there should be a general framework for both the spectral theory of locally symmetric spaces and the many body problem in mathematical physics, and a general theory of analysis on manifolds with corners will be useful for such purposes, since the Borel-Serre compactification is a manifold with corners. He also told me that he had a general procedure of how to self-glue a compact manifold with corners into a closed manifold, which is exactly what we needed. When I told this result to Prof. Borel, he commented that it is amazing that such a topological result was discovered by an analyst.

3. Experiences in Hong Kong. During the visit at IAS in November 1997, Professor Borel told me that Professor Mok at University of Hong Kong had invited him to organize a three year program on Lie theory starting in 1999 when the new Mathematical Research Institute was established. Upon his recommendation, I was invited by Professor Mok to visit University of Hong Kong in May 1998 to give some lectures as preparation for the Borel program. I was also invited to give lectures at the Borel program in 1999 and 2000. Besides mathematics, we talked about many things.

I remember that in the summer of 2000 , he talked enthusiastically about a trail on an off-island not listed in guidebooks and offered to take us there. During one free day, several of the lecturers together with many students followed him to the trail. The trail is on a steep hill, but Prof. Borel walked steadily up, and we young people lagged behind. Dan Barbasch commented that Borel was like a machine: Once his speed was set, it would not decrease. On the top of the hill, we could see very green trees and blue water. He commented that the landscape of Hong Kong was so well preserved that it was like Switzerland in 1950. We also gave a non-mathematical quiz to the Chinese students at the lunch on this trip. He told me earlier that he liked 
Chinese arts. Since China has a long history with so many dynasties, and events are often recorded using the dynasty names, it is not always easy to know precisely the dates. He said that for simplicity, he remembered the years of all the Chinese dynasties in the western calender. It was truly amazing to me, and I told him that I could not do it. I also said that it is not just I who could not remember all these years. This topic came up again on the trip, and I asked him to test the students about this. The result was expected.

His knowledge about the Chinese culture is widely ranged. He told me that on his third trip to China, he joined a group from Beijing to visit the famous Dunhuang Caves on the silk road. A guide from Beijing travelled with the group. Since he knew more than the guide, he often took over and explained the history and paintings in the caves in more detail. The director of the Museum of Dunhuang Caves was so impressed by Prof. Borel's knowledge about the caves that he opened for Prof. Borel some special ones reserved only for experts.

4. Lecture series in Marseille-Luminy and the book project. In July 2001, Prof. Borel and I was invited by J.P.Anker to give a series of lectures on Compactifications of symmetric and locally symmetric spaces at the European Summer School on Lie Theory held at CRM, Marseille-Luminy. It was a wonderful summer school.

Each day there was a long break between the last lecture and the dinner, and I always went to the Mediterranean Sea to swim. Prof. Borel came a few days after the school started. One day I took him to the sea. It was windy and cold. He decided not to swim. But I wanted to go in any case. He thought it is dangerous and tried to persuade me not to go without success. Finally, he said that he did not want to finish the joint book alone.

The joint book project was proposed by N.Wallach near the end of the summer school. In fact, Wallach was also a lecturer of the summer school. He told us that he was an editor of Birkhäuser and suggested us to write a book on compactifications of symmetric and locally symmetric spaces. It is sad to say that I have to finish the book alone.

On an excursion during the summer school, we visited an island next to an island in the harbor of Marseille which was the prison described in the famous novel The count of Monte Cristo by A.Dumas. Prof. Borel told me that this was one of favorite novels. The prison is now a major tourist attraction in Marseille. He said that one way to make it more attractive is to follow the plot of the novel and to put adventurous tourists into sacks tied with stone, to throw them into the sea and then to pull them out; he added that for extra money, the tourists could stay in the water for longer periods.

5. The summer school program in Hangzhou. In March 2002, Prof. Yau suggested me to invite Prof. Borel to co-organize a multi-year program on Lie groups and automorphic forms at the newly established Center of Mathematical Sciences at Zhejiang University. Prof. Borel responded positively and actively to this proposal. We started immediately to discuss detailed plans for this program. He believed that it is important to run a basically self-contained program, starting from basic courses and leading students to research topics. At the IAS/Park City Math Institute in July 2002, Prof. Borel, D.H.Jiang and myself started to write up a proposal for the program and the introduction on the poster. Prof. Borel read and checked every word and sentence and thought them over several times. 
The first part of this summer school at the Center of Mathematical Sciences was scheduled to start in June 2003. By April 2003, everything including the lecture schedule was arranged, and Prof. Borel also prepared some lecture notes. Unfortunately, the SARS epidemic broke out in China and seemed to be out of control, and the program as planned was cancelled. In the middle of May, I started to organize a scaled down summer school with different speakers to begin on August 4, 2003, since I believed that Hangzhou would not be affected by SARS.

Near the end of June, Prof. Borel told me that he had some back pain. One day, his secretary Elly asked me to call Prof. Borel at home about something important. I called and he told me that he had lung cancer. Since I did not know much about cancer and differences of different types and I have heard and known that many people have survived cancer, I thought that it could be controlled since he could get very good care in Princeton. Besides he had always been strong and healthy. On July 31, the day before I left for China for the summer school, I called him. He was still concerned about the summer school in Hangzhou. We agreed that when I came back on August 24, I would call him to let him know how the summer school went, and we could then discuss the plan for the summer school in 2004. He told me that I should be realistic that he might not be able to go to the summer school in 2004 and to give full hour lectures, since at the moment, he could only stand up and give lectures for half an hour. Sadly, it was the last conversation we had.

In the morning of August 11, 2003, when I logged into my email account, I found two messages about the unexpected death of Prof. Borel, one from M.Goresky and another from G.Prasad. It was truly surprising and sad to me. Before I went to China, we talked about the famous Dragon Well green tea from Hangzhou. On Sunday, August 10, 2003, I just went out and bought some green tea for him.

In 2004, the summer school was held in Hangzhou as basically planned Prof. Borel, and was followed by a week long international conference in memory of him.

6. Miscellaneous comments. As mentioned earlier, Prof. Borel has given me advice on health. He also told me how he organized conferences and seminars. He said that the key point is to tell the speakers precisely what was expected. This was also confirmed by W.Casselman. This advice has been very useful to me during the time when I was editing this special issue and when I organized the international conference in memory of him, Algebraic groups, arithmetic groups, automorphic forms and representation theory, July 26-30, at the Center of Mathematical Sciences, Zhejiang University, and the summer schools.

Though he appeared to be serious and unfriendly as many people thought, Prof. Borel was actually a very warm person. During a visit to Ann Arbor, my family went out to dinner with him and his wife Gaby. My second daughter did not like the sweet pea in fried rice and picked it out one by one. Prof. Borel suddenly became very happy and said that he was glad to find someone with the same taste since he did not like sweet pea since childhood. During the dinner, I could clearly see that he began to like my second daughter. Before my children left with my wife in her car, he smiled and tried but hesitated to say goodbye to my second daughter. I can still vividly remember his expression. My children were still young (the second daughter was five years old that time), but they remember Prof. Borel and his wife well and still talk about them now. When I drove them back to the hotel that evening, we talked about children. He said that he would like to have some grandchildren. Once I asked him if he helped raise his children when they were babies. He said that he stayed home for some time after his daughters were born, read and followed precisely the advice of 
some standard book on raising children.

On many occasions, when I met him, it was a smile on his face that I first saw. This will be the picture I will remember of him. 
L. JI 